\title{
Predictive Ability of a Laboratory Performance Test in Mountain Bike Cross-country Olympic Athletes
}

\author{
Authors \\ Patrick Schneeweiss ${ }^{1}$, Philipp Schellhorn ${ }^{1}$, Daniel Haigis ${ }^{1}$, Andreas Niess ${ }^{1}$, Peter Martus², Inga Krauss ${ }^{1}$
}

Affiliations

1 Department of Sports Medicine, Medical Clinic, University of Tuebingen, Tuebingen

2 Institute for Clinical Epidemiology and Applied Biometry, University of Tuebingen, Tuebingen

\section{Key words}

off-road cycling, performance testing, mountain biking, competition, validity, XCO

\section{accepted 06.02.2019}

\section{Bibliography}

DOI https://doi.org/10.1055/a-0858-9900

Published online: 1.4.2019

Int J Sports Med 2019; 40: 397-403

(c) Georg Thieme Verlag KG Stuttgart · New York

ISSN 0172-4622

\section{Correspondence}

Mr. Patrick Schneeweiss

Department of Sports Medicine, Medical Clinic,

Hoppe-Seyler-Str. 6,

72076 Tuebingen,

Germany

Tel.: + 49/707/12986 496, Fax: + 49/707/12925028

patrick.schneeweiss@med.uni-tuebingen.de

\begin{abstract}
Mountain bike Cross-Country Olympic (XCO) has an intermittent performance profile, underlining the importance of anaerobic metabolism. Traditional performance tests in cycling primarily quantify aerobic metabolism and inadequately meet the demands in XCO. The aim was therefore to validate a specific test that quantifies these requirements by means of an XCO race. Twenty-three competitive XCO athletes ( $17.9 \pm 3.6$ years) performed a previously developed performance test and an XCO race within one week. Correlations between individual anaerobic threshold (IAT), 4 mmol lactate threshold $\left(\mathrm{LT}_{4}\right)$, maximal aerobic power (MAP), maximal effort time trials (TT) for 10-300 s and mean power output of the race (POR) were calculated. In addition, a multiple regression model of the predictive value of the test was calculated. Variables correlated significantly $(p<.01)$ with POR: IAT $(r=.81), \mathrm{LT}_{4}(r=0.79), \mathrm{MAP}(r=0.91), \mathrm{TT}_{10}$ $(r=0.75), \mathrm{TT}_{30}(r=0.85), \mathrm{TT}_{60}(r=0.84)$ and $\mathrm{TT}_{300}(r=0.86)$. In the regression model, sex and body mass were set influencing variables $\left(R^{2}\right.$ adj. $\left.=0.70\right)$, whereby MAP had the highest correlation with POR and significantly improved the predictive value of the model $\left(R^{2}\right.$ adj. $\left.=0.86\right)$. The high correlation of collected performance variables with POR indicated the MTB-PT's additional benefit for performance testing in XCO because it is specific but very feasible.
\end{abstract}

\section{Introduction}

Mountain bike Cross-Country Olympic (XCO) is one of the main disciplines of mountain biking (MTB). The race has a mass start, with all of the athletes starting at the same time. Athletes have to finish 4-6 laps on the course, which is about $5 \mathrm{~km}$ long, leading to race durations from 80 up to $100 \mathrm{~min}$. This cycling event is considered a high-intensity intermittent activity because of its multitude of alternating climbs and downhill sections [4, 13, 16, 27]. Exercise intensities may further vary according to race course characteristics $[6,27]$. These high-intensity demands have been expanding lately because race duration has been shortened, race profiles have become more irregular, and technical demands of the courses continuously increase [29, 30]. Recent data on adolescent competitive XCO athletes showed that 3-min intervals at $120 \%$ of the individual anaerobic threshold (IAT; as defined by Dickhuth et al. [7] and Roecker et al. [26]) and shorter intervals of approximately $20 \mathrm{~s}$ at
$240 \%$ IAT are particularly frequent in XCO-MTB [29]. Yet, exercise training is inspired by power-based training levels and modified high-intensity training zones to account for the short- and medium-term high-load events, especially of XCO races [3, 29].

These physiological demands should also be mapped in the context of exercise performance testing to improve training and to predict race performance, especially in competitive athletes [28]. For this purpose, a performance test should comprehensively cover the physiological demands of the sport and sufficiently predict race performance. The traditional approach to predict race performance in cycling is an incremental laboratory test on a cycle ergometer (graded exercise test, GXT). Although previous studies have indicated that a GXT predicts mountain bike race performance sufficiently, its ability to explain the variance of race performance differs from 44 to $92 \%$ [14, 15, 23]. Therefore, Prins et al. [23] described the need for a better tailored test design and suggested a shift to- 
wards less traditional laboratory tests that consider the demands of MTB. In recent years, more comprehensive approaches using both aerobic (GXT) and anaerobic (short time trials) tests have adopted multiple variables within performance analyses [16, 19, 21, 23]. Inoue et al. [16] investigated the relationship between XCO performance and measures taken from a GXT and several Wingate tests. In contrast to Prins et al. [23], this study used a typical mass-start race to assess the XCO performance and did not include individual time-trials. Maximal aerobic power (MAP; $r=0.79$ ) and mean power during repeated Wingate tests $(r=0.89)$ were strongly correlated with XCO race performance. In addition, Novak et al. [21] applied a multidimensional approach including a decision-making test that strongly predicted performance within $X C O\left(R^{2}=0.92\right)$. These and previous findings highlight the influence and importance of anaerobic skills on XCO race performance [16]. However, authors conclude that their approach may be less practical because of the timeconsuming test procedure lasting several days.

As a consequence, Ahrend et al. [2] conducted a pilot study to validate a comprehensive test battery that comprised a GXT, simulated time trials (TT; maximal efforts with $10 \mathrm{~s}, 60 \mathrm{~s}$ and $300 \mathrm{~s}$ duration) and maximal isometric strength testing. The chosen durations of TT were considered to be typical in XCO and are often used for training purposes and diagnostics [3, 13, 24, 27]. The findings of this pilot study underlined that mainly aerobic parameters of the GXT as well as power output during the short high-intensive time trials should be considered when analysing performance of XCO athletes. Subsequently, Ahrend et al. examined the predictive ability of a specific performance test in mountain bike marathon (XCM) [1]. The laboratory test included both traditional variables (IAT, MAP) and simulated time trials. It was feasible within $1 \mathrm{~h}$ and thus with considerable reduced time commitment for the athlete. The multiple regression models explained $87 \%$ of the race time variance for a single XCM race and $76 \%$ of several races after a z-transformation, demonstrating significant validity. However, these findings were made at XCM and do not necessarily have to be valid for XCO as well.

Referring to these findings, the present study aimed to determine the predictive ability (criterion validity) of an MTB-specific performance test in XCO athletes. We hypothesised that the predictive ability of this MTB specific performance test (MTB-PT) outperforms the predictive ability of the traditional graded exercise tests.

\section{Materials and Methods}

\section{Participants}

Recruitment of eligible female and male participants occurred via trainers, clubs, and personal contacts within the MTB community. After

- Table 1 Classification of XCO athletes (mean $\pm S D ; n=23$ ).

\begin{tabular}{|l|l|l|l|l|l|}
\hline & $\mathbf{n}$ U17 & $\begin{array}{l}\mathbf{n} \text { U19, } \\
\text { U23, } \\
\text { Elite }\end{array}$ & $\begin{array}{l}\text { n race } \\
\text { (a) } \\
\text { (4 laps) }\end{array}$ & $\begin{array}{l}\text { n race } \\
\text { (b) } \\
\text { (6 laps) }\end{array}$ & $\begin{array}{l}\text { age } \\
\text { [years] }\end{array}$ \\
\hline Women & 2 & 4 & 6 & 0 & $16.8 \pm 1.8$ \\
\hline Men & 6 & 11 & 6 & 11 & $18.3 \pm 4.0$ \\
\hline Total & 8 & 15 & 12 & 11 & $17.9 \pm 3.6$ \\
\hline
\end{tabular}

U17/U19/U23: denotes athletes under $17 / 19 / 23$ years of age a telephone screening, 30 athletes were invited for a first visit. Of those, 23 XCO athletes were finally included in the study ( $\triangleright$ Table 1). They competed in national junior classes or the elite class in the current season. According to the respective age group, the experience of the athletes regarding training and competition was typical for the national competition class. However, the extent of experience with training and competitions was unfortunately not systematically recorded.

Due to a technical defect and the consequences of a slight cold, 2 athletes could not finish the XCO race. In the end, 21 data sets were analysed completely. All participants provided written informed consent and were screened for medical contraindications for exercise by a medical doctor. Ethical approval was received from the local ethics committee (number 472/2016BO1) and the study was registered in the national database (number PR020160800134) and meets the ethical standards of the journal [11].

\section{Procedures}

After the medical examination was conducted and anthropometric measures were taken, all athletes performed a previously developed mountain bike-specific performance test (MTB- PT) [1, 2] and a simulated XCO race within one week. Athletes were instructed to avoid strenuous exercise, alcohol and other drugs for at least $24 \mathrm{~h}$ prior to the laboratory tests and the simulated race; beyond that they were instructed to maintain their usual preparation for competition. Furthermore, long-term medication was not allowed and was checked by a questionnaire and during the medical examination by the medical doctor.

\section{MTB-specific performance test (MTB-PT)}

A standard MTB was mounted on a Cyclus2 ergometer (RBM elektronik-automation, Leipzig, Germany) and adapted to the specific requirements of the athlete (seat post, handle bar, pedal system). The elastic suspension of the Cyclus2 ergometer allows lateral oscillations, which makes the laboratory test feel more like riding a real MTB. The bike was equipped with an SRM training system consisting of an SRM Powermeter (instrumented crank) and the SRM Powercontrol (data logger and on-board data display; PC8) (Schoberer Rad Messtechnik, Welldorf, Germany). Due to its high validity, reliability and sensitivity, the SRM is considered the gold standard for mobile power meters [8, 22]. The athlete's cycling power output, cadence (pedalling frequency), and heart rate were continuously recorded at $1 \mathrm{~Hz}$ by the PC8 via ANT + . Mean values for all time trials were automatically calculated by the free GoldenCheetah training software (www.goldencheetah.org; version 3.3).

The MTB-PT ( Fig. 1) started with a graded exercise test (GXT) at an initial load of 80 watts $(W)$. The workload was increased every 3 min by $40 \mathrm{~W}$ until self-imposed exhaustion. In order to quantify the maximal exertion, the individually achieved highest heart rate at GXT ( $\left(H R_{\mathrm{GXT}}\right)$ was compared with the athlete's maximal heart rate during the whole MTB-PT and the race $\left(\mathrm{HR}_{\max }\right)$. Furthermore, blood lactate concentration at the end of the GXT was evaluated. During the GXT athletes were advised to maintain a cadence of at least 60 revolutions per minute (rpm). The MAP was calculated using the equation by Kuipers et al. [17]:

$M A P=W_{f}+(t / 180 \times 40)$ 


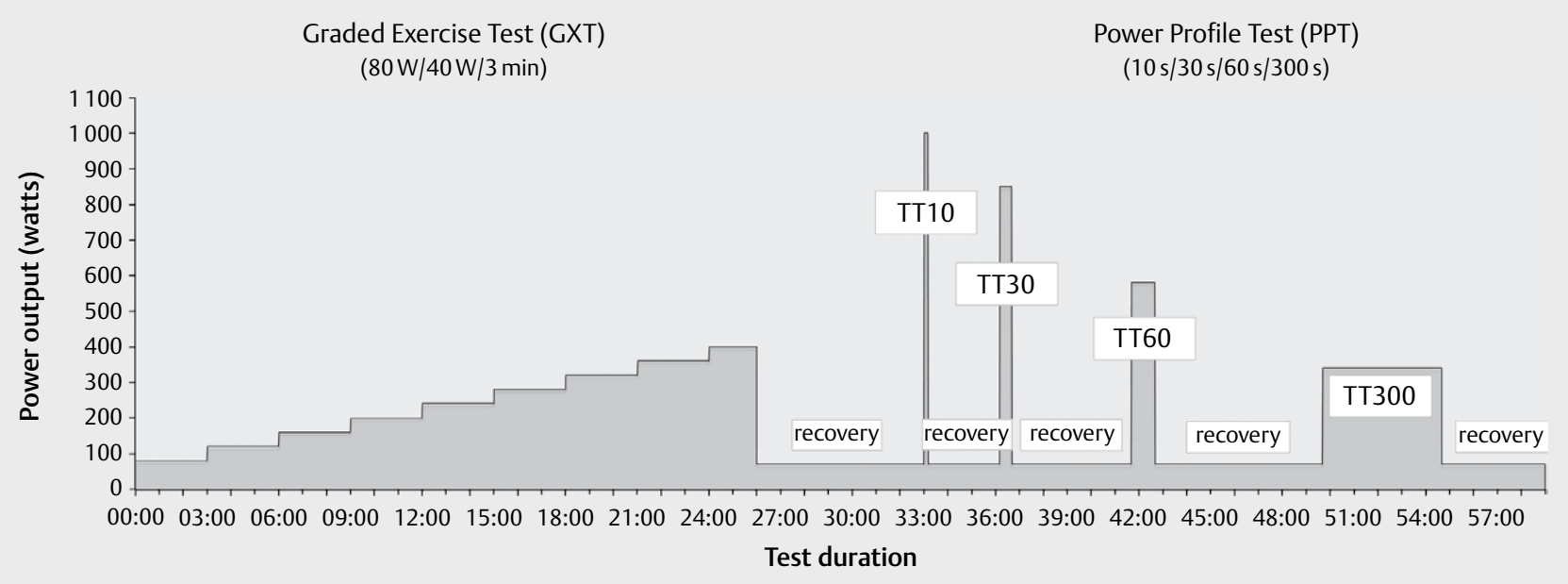

- Fig. 1 MTB-specific performance test (MTB-PT). TT10-300 = time trials lasting 10 to 300 s (sprint/maximal effort); recovery= period of recovery at $1.2 \mathrm{~W}^{*} \mathrm{~kg}^{-1}$ body mass.

where $\mathrm{W}_{\mathrm{f}}$ was the last completed workload, and $\mathrm{t}$ is the time in seconds of the uncompleted workload.

After terminating the GXT, athletes continued pedalling and proceeded with a Power Profile Test (PPT). It comprised: i) a 7-min recovery period followed by a 10 -s all-out sprint $\left(\mathrm{TT}_{10}\right)$; ii) a 3-min recovery period followed by a 30 -s all-out sprint $\left(\mathrm{TT}_{30}\right)$; iii) a 5 -min recovery period followed by a 60 -s maximal effort ( $\mathrm{TT}_{60}$ ); iv) a 7 -min recovery period followed by a 300 -s maximal effort ( $\left.\mathrm{TT}_{300}\right)$; and $\mathrm{v}$ ) a final 5-min recovery period. During recovery periods, the athletes were asked to pedal at a power output of $1.2 \mathrm{~W}$ per kilogram of body mass. As the PPT was driven in the simulation mode of the Cyclus2 ergometer, athletes were allowed to complete the PPT with their self-selected cadence and in a seated or standing position. In order to prevent pacing and ensure that maximal effort was performed, strong verbal encouragement was given by the test instructor.

Blood lactate was analysed (Biosen S-Line, EKF, Cardiff, UK) by collecting capillary blood samples $(20 \mu \mathrm{L})$ from the right earlobe during the last $20 \mathrm{~s}$ of each stage of the GXT. The IAT was defined as the cycling power output at a lactate concentration of $1.0 \mathrm{mmol}^{*} \mathrm{~L}^{-1}$ above the lowest lactate to power output quotient that describes the onset of lactate increase as proposed by Dickhuth et al. [7] and Roecker et al. [26].

\section{Simulated XCO-MTB race}

The 2 races were organized for the study participants only and carried out on a slightly modified official Union Cycliste Internationale (UCI) XCO race track in Albstadt, Germany. One lap of the modified track with $130 \mathrm{~m}$ ascending elevation was about $2100 \mathrm{~m}$ long and started 750 meters above sea level ( $>$ Fig. 2 ). The weather conditions were very good: the track was almost dry, there was no rain or wind, and the mean air temperature was about $14^{\circ} \mathrm{C}$.

To account for age and sex differences of the given sample, races were conducted separately for (a) female athletes and male athletes younger than 17 years with 4 laps and (b) male athletes at least 17 years old with 6 laps ( $\triangleright$ Table 1 ).

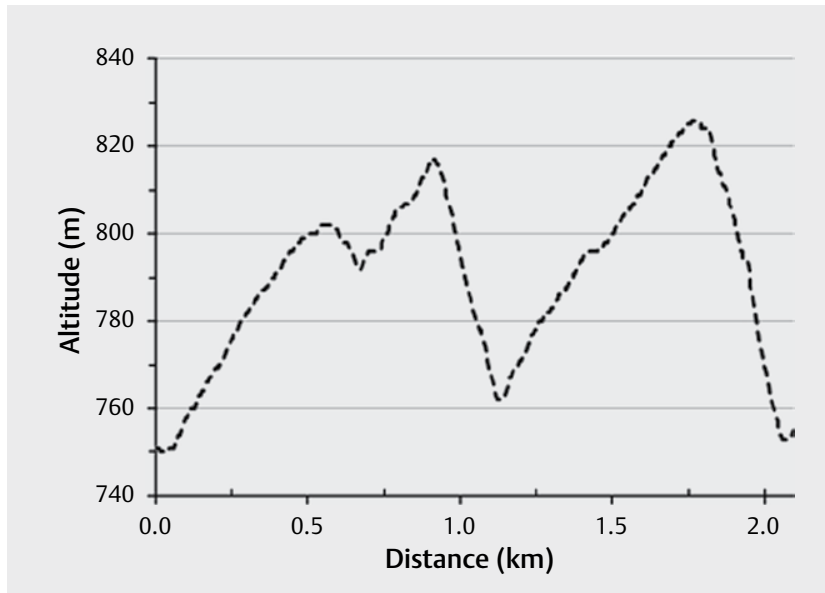

- Fig. 2 Track profile of one lap of the XCO course that has been driven 4 (Race a) or 6 times (Race b).

The original crank sets on the athletes' mountain bikes were replaced by SRM training systems to achieve the best possible comparability between the laboratory and field measures. The athletes were encouraged to complete the race as fast as possible. In order to avoid disadvantages due to starting position, the athletes were positioned by the coaches in 2 starting rows according to their previous racing performance. The athlete's power output (including the zero values), cadence, heart rate and location/altitude were continuously recorded at $1 \mathrm{~Hz}$ by the PC8. Additionally, the lap times were recorded. After the race, athletes were asked about possible race disturbances and interruptions, such as falls or technical problems. Athletes with serious technical problems or injuries during the races were excluded. 


\section{Statistical analysis}

Mean power output of the race (POR) was used as a criterion variable for race-specific validation of the laboratory variables or the MTB-PT.

Descriptive results are presented as mean \pm SD. Pearson's correlation coefficient $r$ was used for the quantitative measure of the correlation between each laboratory variable and POR. The coefficient was interpreted using a categorization proposed by Hinkle et al. [12]: 0.9-1.0 very high, 0.7-0.9 high, 0.5-0.7 moderate, 0.30.5 low, 0.0-0.3 negligible correlation. Additionally, we have listed $R^{2}$ and for multiple regression models the adjusted $R^{2}\left(R^{2}\right.$ adj.) as a statistical measure of model fit. In the multiple regression analysis, sex and body mass were entered in the first model step; in the second step forward variable selection was applied (probability for inclusion $=0.05$, exclusion $=0.10)$. The standard error of the estimate (SEE) was calculated as the square root of the residual mean square. Data were analysed with IBM SPSS Statistics v.23.0 (IBM Corp, 2015). The level of significance was $\alpha=0.05$ (2-sided) in all statistical testing.

In order to obtain a more appropriate measure of the athlete's performance, the power output data was additionally normalized with the athlete's body weight. This is a common technique and allows a better assessment of the athlete's individual power output performance so that training recommendations can be derived $[10,13]$.

\section{Results}

The anthropometric and performance characteristics of all athletes are summarized in > Table 2.

The athlete's individual $\mathrm{HR}_{\max }$ was $196 \pm 8 \mathrm{bpm}$ compared to $196 \pm 8$ bpm after the GXT $\left(H_{\mathrm{GXT}}\right)$ and thus almost identical. Blood lactate concentration at the end of the GXT was $11.5 \pm 2.1 \mathrm{mmol}^{*} \mathrm{I}^{-1}$ and ranged from 8.9-15.9 $\mathrm{mmol}^{*} \mathrm{I}^{-1}$.

All diagnostic power output variables showed high to very high and statistically significant correlations with race performance (POR): IAT ( $r=0.81), \mathrm{LT}_{4}(r=0.79), \operatorname{MAP}(r=0.91), \mathrm{TT}_{10}(r=0.75), \mathrm{TT}_{30}$ $(r=0.85), \mathrm{TT}_{60}(r=0.84)$ and $\mathrm{TT}_{300}(r=0.86)$. For further details, see Fig. 3. Moreover, the several diagnostic variables showed large intercorrelations from $r=0.84$ to $r=0.99$ (results not given in detail).

In the regression model, the entered predictors sex and body mass explained $70 \%$ of the variance $\left(R^{2}{ }_{\text {adj }}=0.70\right.$; $\left.S E E=25.2\right)$ concerning the dependent variable POR. The model comprised MAP as the only variable that significantly improved the predictive power of the model. All other physiological performance variables were excluded because they could not contribute significantly to improving the fit of the model. Thus, $86 \%\left(R^{2}\right.$ adj $=0.86$; $\left.\mathrm{SEE}=17.2\right)$ of the variance of POR could be explained with the entered variables sex and body mass and the predictor MAP. The check of the residual plots revealed no residual patterns that could indicate biased results.

\section{Discussion}

This study aimed to determine the predictive ability (criterion validity) of an MTB-specific performance test in 23 XCO athletes by correlating laboratory variables with the mean power output in an XCO race. However, the main hypothesis that the MTB-PT is supe-

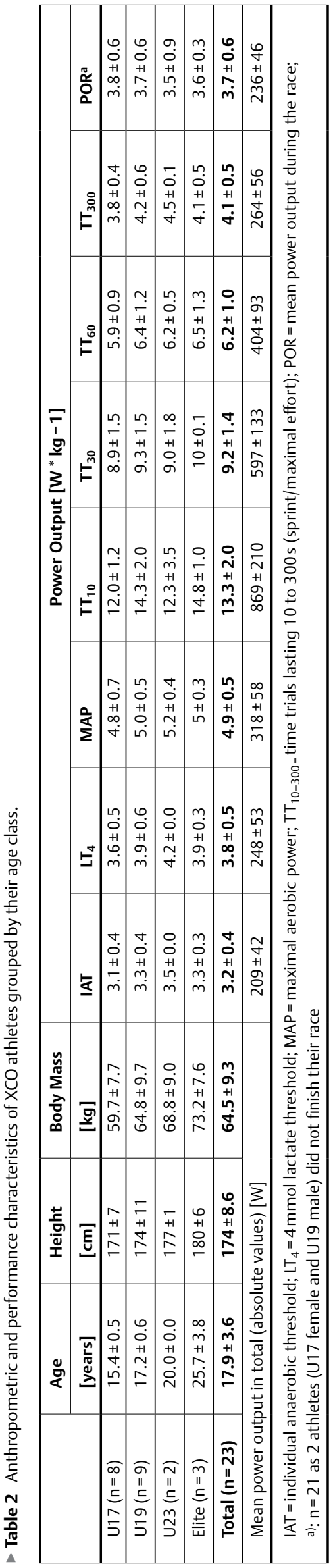



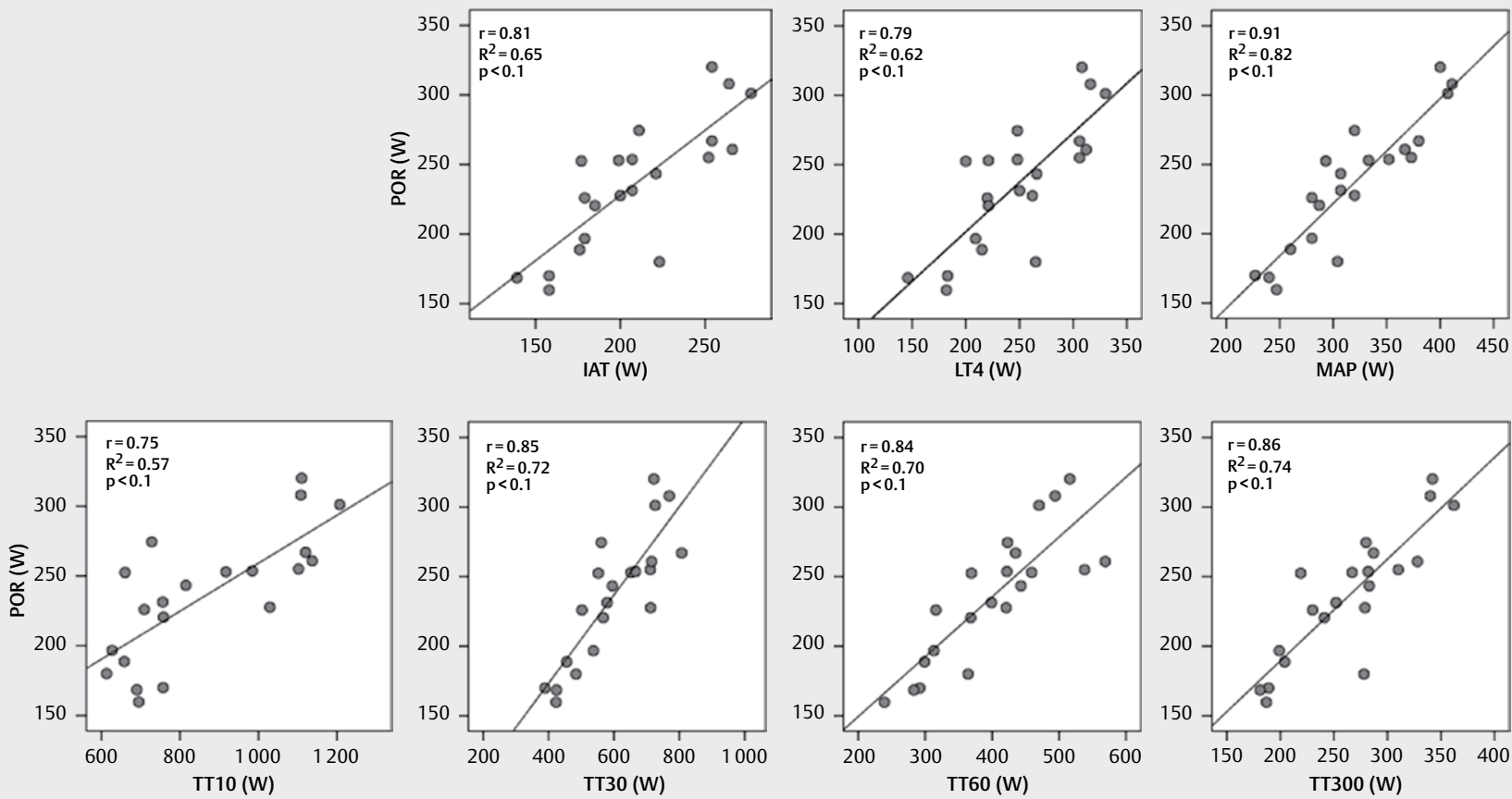

- Fig. 3 Linear correlations of mean power output during the race $(P O R)$ with laboratory variables. $P O R=$ mean power output during the race; IAT = individual anaerobic threshold; LT_4 $=4 \mathrm{mmol}$ lactate threshold; MAP = maximal aerobic power; TT10 $-300=$ time trials lasting 10 to $300 \mathrm{~s}$ (sprint/maximal effort); $(n=21)$.

rior to the GXT could not be statistically confirmed, because the multiple regression contained MAP as the only variable that significantly improved the predictive power of the model. Nevertheless, the MTB-PT is superior in terms of practical applicability as individual diagnostic values provide feasible reference values for training purposes.

Some other authors concluded that their approach of predicting cycling race performance may be less practical because of the time-consuming test procedure lasting several days. In contrast to most other studies, this study used a laboratory test protocol that took only about an hour to complete. Thus it is also very feasible for all interested athletes, even outside of study settings. As previously mentioned, the MTB-PT consisted of a GXT, followed by a physiologically demanding MTB-specific test (PPT), which aimed to further increase the predictability of exercise performance in an XCO race in comparison to the GXT only. The GXT was included due to its previously reported association with XCO performance (IAT and MAP). The PPT was added to quantify cycling performance during XCO-typical load periods of $10-300$ s $[3,13,27]$. Especially during very high-intensity and maximal efforts (as demanded by PPT), exercise capacity may also be limited by the amount of energy obtained from the anaerobic energy storage [5]. Nevertheless MAP, similar to maximal oxygen uptake ( $\mathrm{VO}_{\text {max }}$ ), is one of the most important physiological variables correlated with endurance and maximal performances [16]. Maximal heart rate is widely used as a criterion for achieving peak exertion and therefore the opportunity to determine MAP [18]. Given the fact that in highly trained athletes, the widely employed formula $\mathrm{HR}_{\max }=220$ - age significantly overestimates the age-predicted maximal heart rate [31],
$\mathrm{HR}_{\mathrm{GXT}}$ was compared with the athlete's $H R_{\max }$ during the whole MTB-PT and the race. With the exception of one athlete, all athletes reached their $\mathrm{HR}_{\max }$ during the GXT, indicating exhaustion of all athletes after the GXT, which was confirmed by the lactate concentrations ranging from 8.9 to $15.9 \mathrm{mmol}^{*} \mathrm{I}^{-1}$.

The variables based on lactate threshold concepts showed large variability $(r=0.3-0.9)$ between previous studies $[6,15,23]$, indicating the difficulty to predict race performance in mountain biking via the GXT. We determined the $4 \mathrm{mmol}$ lactate threshold $\left(\mathrm{LT}_{4}\right)$ because power output at $\mathrm{LT}_{4}$ is quite close to the functional threshold (FTP) [9]. This should add valuable practical implementation opportunities for power based training, as training recommendations are often based on FTP [3].

In the present study, the GXT outcomes showed high to very high correlations and could explain the race performance of the XCO race with 65,62 and $82 \%$ for IAT, $\mathrm{LT}_{4}$, and MAP, respectively. Previous studies have confirmed that $\mathrm{VO}_{\text {max }}$ and MAP correlated strongly $(r=0.6-0.9)$ with performance in XCO competition $[2,10$, $15,23]$. Nonetheless, a large fraction of the variance in performance remains unexplained. Impellizzeri et al. [14] investigated 12 international-level athletes and reported correlations between MAP and race time of $r=-0.48$. In contrast, Costa and De-Oliveira [6] found a very high correlation between MAP and rank position in 2 races $(r=-0.88$ and $r=-0.88)$. Ahrend et al. [1] investigated 49 competitive mountain bike riders and reported correlations between MAP and race time of 3 XCM races with $r=-0.77, r=-0.73$ and $r=-0.76$, respectively. 
Similar to the results of a previous study with a comparable setting [2], the correlations of the different TT with race performance (POR) were higher than those of the GXT outcomes, except for MAP. Whereas $\mathrm{TT}_{30}, \mathrm{TT}_{60}$, and $\mathrm{TT}_{300}$ showed high correlation with $\mathrm{POR}$, the correlation of $\mathrm{TT}_{10}$ was somewhat weaker. The variance of racing performance can be explained by $57 \%\left(\mathrm{TT}_{10}\right), 72 \%\left(\mathrm{TT}_{30}\right), 70 \%$ $\left(\mathrm{TT}_{60}\right)$, and $74 \%\left(\mathrm{TT}_{300}\right)$, respectively. With regard to the lower correlation of $\mathrm{TT}_{10}$ with the POR, it should be noted that the informative value of the $\mathrm{TT}_{10}$ in this form is not convincing. However, given the predictive power of the other time trials, the $\mathrm{TT}_{10}$ must be maintained, because the time trials influence each other with regard to a cumulative fatigue effect. In the multiple regression model, the entered predictors sex and body mass explained $70 \%$ of the variance in POR. It is obvious that sex and body mass are influencing factors for power output in mountain bike racing, as previous investigations have shown [1]. The model further comprised MAP as the only variable that significantly improved the predictive power of the model. This is in contrast to our expectations based on previous findings in which multiple regression models explained $87 \%$ of the race time variance for a single XCM race and $76 \%$ of several races after a z-transformation by using $\mathrm{TT}_{60}$, IAT, and body weight [1]. Despite these differences in input variables, the present results are comparable with respect to their predictive power. Eighty-six percent $\left(R^{2}\right.$ adj. $=0.86$; $\left.S E E=17.2\right)$ of the variance in POR could be explained with the entered variables sex and body mass and the predictor MAP. Due to the limited sample size and the high correlation of potential predictors, we cannot exclude the possibility that additional predictors different from MAP may contribute significantly to the prediction of race performance. This has to be explored in larger studies. It should also be mentioned that in explorative calculations, the regression model also showed a good fit for the predictor $\mathrm{TT}_{300}$ and the entered variables sex and body mass $\left(R^{2}\right.$ adj. $\left.=0.83\right)$. A slight improvement of $1.6 \%$ could be achieved by adding $\mathrm{TT}_{30}$ to the entered variables sex and body mass and the predictor MAP. Due to the limited sample size, the relevance of $\mathrm{TT}_{30}$ and $\mathrm{TT}_{300}$ for predicting race performance cannot be excluded. Based on our data with only 6 women, we cannot conclude whether separate models for men and women should be chosen. However, we recommend exploring this if the sample size for both sexes is sufficiently large.

The explanatory power of this model is slightly smaller than the explanatory power described for laboratory tests to predict a $10 \mathrm{~km}$ run (89\%), half marathon (92\%), and marathon (90\%) with 3 independent variables [25]. In contrast to a running competition with a predefined distance and negligible differences in altitude or running surfaces, XCO races can differ remarkably with respect to physiological and technical demands as well as environmental conditions (e. g., wet, slippery ground; rain). This could explain the comparatively lower clarification of the total XCO performance by physiological variables. Due to these limitations on the comparability of the results with those of other tests focusing on short- and medium-term intervals, they cannot be directly compared with previously published results. Costa and De-Oliveira [6] found only small and non-significant correlations between $\mathrm{TT}_{30}$ (normalized by body mass) and the rank position in 2 XCO races ( $r=-0.12$; $r=-0.29$ ). Inoue et al. [16] investigated 10 XCO athletes performing 5 Wingate anaerobic tests (WAnT) with a 30-s recovery bet- ween the repetitions. The mean power output of the 5 WAnT normalized by body mass correlated significantly with race time $(r=-0.63 ; P<0.05)$. Several factors can make it troublesome to compare our results with those of other studies. Differences in the correlation coefficients between the studies could also be explained by differences in sample size and study populations as well as by the use of absolute vs. body mass normalized power output values. In addition, different lactate threshold concepts and differences in the test protocol design can influence the correlations. However, the present results tend to show higher correlations than previous studies. The individual values thus provide feasible reference values for training purposes, as they can be directly associated with the physiological requirements in XCO races. In powerbased training, the respective training targets can be regulated and the success can be controlled by the gained findings.

It should be undisputed that the GXT ahead of the PPT influences the power output during the maximal efforts because athletes cannot completely recuperate during the recovery periods. By reason of increasing fatigue over time, the power outputs of the sprints and maximal efforts are expected to be smaller than those seen without preloading. The anaerobic power is important for XCO cyclists, but with each repeated effort of high intensity they rely more and more on their aerobic system, indicating the need to have a welldeveloped aerobic system, too. However, this series of load peaks in the absence of adequate regeneration corresponds to the requirements in XCO races. Other important factors that influence the overall performance in XCO racing are certainly the technique and tactics of the athletes and their handling of the given external conditions. The terrain, course, and weather conditions, for example, could also have a determining influence. However, these factors cannot be measured with a practicable and easy-to-standardise laboratory diagnostic method.

In conclusion, the findings of this study confirm past research suggesting that XCO performance is based on a combination of highly developed aerobic and anaerobic capacities as well as skillbased characteristics $[14,16,19,20,23]$. Nevertheless, the findings suggest that laboratory data may provide an accurate means of modelling cycling performance. Whether our model can be used to predict performance on other XCO tracks will need further investigation.

Due to its short time expenditure of only about $1 \mathrm{~h}$, the MTB-PT is very suitable for repeated measurements to render objective performance and training success during the course of a season and has a strong practical application for both XCO trainers and cyclists.

\section{Acknowledgements}

We would like to thank all the volunteers who took part in this study, which was funded by the Federal Institute for Sports Science (BISp).

\section{Conflict of Interest}

Authors declare that they have no conflict of interest. 


\section{References}

[1] Ahrend M-D, Schneeweiss P, Martus P, Niess AM, Krauss I. Predictive ability of a comprehensive incremental test in mountain bike marathon. BM] Open Sport Exerc Med 2018; 4: e000293

[2] Ahrend M-D, Schneeweiss P, Theobald U, Niess AM, Krauss I. Comparison of laboratory parameters of a mountain bike specific performance test and a simulated race performance in the field. J Sci Cycling 2016; 5: 3-9

[3] Allen $\mathrm{H}$, Coggan A. Training and Racing with a Power Meter. 2nd ed VeloPress; 2010

[4] Baron R. Aerobic and anaerobic power characteristics of off-road cyclists. Med Sci Sports Exerc 2001; 33: 1387-1393

[5] Capelli C, Rittveger J, Bruseghini P, Calabria E, Tam E. Maximal aerobic power and anaerobic capacity in cycling across the age spectrum in male master athletes. Eur J Appl Physiol 2016; 116: 1395-1410

[6] Costa V, De-Oliveira F. Physiological variables to predict performance in cross-country mountain bike races. J Exerc Physiol Online 2008; 11: $14-24$

[7] Dickhuth HH, Huonker M, Münzel T, Drexler H, Berg A, Keul ]. Individual anaerobic threshold for evaluation of competitive athletes and patients with left ventricular dysfunction. In Bachl TG, Löllgen H. (eds.). Advances in Ergometry. Berlin, Heidelberg, New York: Springer Verlag, 1991: 176-179

[8] Gardner AS, Stephens S, Martin DT, Lawton E, Lee H, Jenkins D. Accuracy of SRM and power tap power monitoring systems for bicycling. Med Sci Sports Exerc 2004; 36: 1252-1258

[9] Gavin TP, Van Meter JB, Brophy PM, Dubis GS, Potts KN, Hickner RC. Comparison of a field-based test to estimate functional threshold power and power output at lactate threshold. I Strength Cond Res 2012; 26: 416-421

[10] Gregory J, Johns DP, Walls JT. Relative vs. absolute physiological measures as predictors of mountain bike cross-country race performance. J Strength Cond Res 2007; 21: 17-22

[11] Harriss D], Macsween A, Atkinson G. Standards for ethics in sport and exercise science research: 2018 update. Int J Sports Med 2017; 38: 1126-1131

[12] Hinkle DE, Wiersma W, Jurs S. Applied Statistics for the Behavioral Sciences, International Edition. 5th ed Andover: Cengage Learning EMEA; 2009

[13] Impellizzeri FM, Marcora SM. The physiology of mountain biking. Sports Med 2007; 37: 59-71

[14] Impellizzeri FM, Marcora SM, Rampinini E, Mognoni P, Sassi A. Correlations between physiological variables and performance in high-level cross-country off-road cyclists. Br J Sports Med 2005; 39: 747-751

[15] Impellizzeri FM, Rampinini E, Sassi A, Mognoni P, Marcora S. Physiological correlates to off-road cycling performance. J Sports Sci 2005; 23: $41-47$
[16] Inoue A, Sa Filho AS, Mello FC, Santos TM. Relationship between anaerobic cycling tests and mountain bike cross-country performance. J Strength Cond Res 2012; 26: 1589-1593

[17] Kuipers H, Verstappen FT, Keizer HA, Geurten P, van Kranenburg G. Variability of aerobic performance in the laboratory and its physiologic correlates. Int J Sports Med 1985; 6: 197-201

[18] Medicine ACoS. ACSM's Guidelines for Exercise. Testing and Prescription. Riebe D, Ehrman JK, Liguori G, Magal M. (eds.) 10th ed Philadelphia: Wolters Kluwer Health; 2018

[19] Miller MC. Validity of using functional threshold power and intermittent power to predict cross-country mountain bike race outcome. J Sci Cycling 2014; 3: 16-20

[20] Miller MC, Macdermid PW, Fink PW, Stannard SR. Performance and physiological effects of different descending strategies for cross-country mountain biking. Eur J Sport Sci 2017; 17: 279-285

[21] Novak AR, Bennett KJM, Fransen J, Dascombe BJ. A multidimensional approach to performance prediction in Olympic distance cross-country mountain bikers. J Sports Sci 2018; 36: 71-78

[22] Paton CD, Hopkins WG. Tests of cycling performance. Sports Med 2001; 31: 489-496

[23] Prins L, Terblanche E, Myburgh KH. Field and laboratory correlates of performance in competitive cross-country mountain bikers. J Sports Sci 2007; 25: 927-935

[24] Quod M], Martin DT, Martin JC, Laursen PB. The power profile predicts road cycling MMP. Int J Sports Med 2010; 31: 397-401

[25] Roecker K, Schotte O, Niess AM, Horstmann T, Dickhuth HH. Predicting competition performance in long-distance running by means of a treadmill test. Med Sci Sports Exerc 1998; 30: 1552-1557

[26] Roecker K, Striegel H, Dickhuth HH. Heart-rate recommendations: Transfer between running and cycling exercise? Int J Sports Med 2003; 24: $173-178$

[27] Stapelfeldt B, Schwirtz A, Schumacher YO, Hillebrecht M. Workload demands in mountain bike racing. Int J Sports Med 2004; 25: 294-300

[28] Stevens C], Dascombe B]. The reliability and validity of protocols for the assessment of endurance sports performance: an updated review. Meas Phys Educ Exerc Sci 2015; 19: 177-185

[29] Theobald U. Leistungsanforderungen und trainingsmittel in der radsportdisziplin mountainbike cross-country. Leistungssport 2015; 1: 20-24

[30] UCl Cycling Regulations. Part 4. Mountain Bike.; URL http://www.uci. $\mathrm{ch} / \mathrm{mm} /$ Document/News/Rulesandregulation/17/29/73/4MTB-E1.01.2018-Final_English.pdf

[31] Whyte GP, George K, Shave R, Middleton N, Nevill AM. Training-induced changes in maximum heart rate. Int J Sports Med 2008; 29: $129-133$ 